\title{
Reconfiguring the scope and practice of regional anesthesia in a pandemic: the COVID-19 perspective
}

\author{
Balakrishnan Ashokka 지, 1,2 Arunangshu Chakraborty (10 , \\ Balavenkat J Subramanian, ${ }^{4}$ Manoj Kumar Karmakar (i) , ${ }^{5}$ Vincent Chan ${ }^{6}$
}

\begin{abstract}
${ }^{1}$ Anaesthesia, National University Health System, Singapore

${ }^{2}$ Centre for Medical Education, National University Singapore Yong Loo Lin School of Medicine, Singapore ${ }^{3}$ Anaesthesia, Critical Care and Pain, Tata Medical Center, Kolkata, West Bengal, India ${ }^{4}$ Anaesthesia, Ganga Medical Centre and Hospitals Pvt Ltd, Coimbatore, Tamil Nadu, India ${ }^{5}$ Department of Anesthesia and Intensive Care, The Chinese University of Hong Kong, Shatin, Hong Kong

${ }^{6}$ Department of Anesthesiology \& Pain Medicine, University of Toronto, Toronto, Ontario, Canada
\end{abstract}

Correspondence to Dr Balakrishnan Ashokka, Anaesthesia, National University Health System, Singapore, Singapore; ashokkab@gmail.com

Received 11 April 2020 Revised 4 May 2020 Accepted 4 May 2020 Published Online First 28 May 2020

\begin{abstract}
The COVID-19 outbreak is on the world. While many countries have imposed general lockdown, emergency services are continuing. Healthcare professionals have been infected with the virulent severe acute respiratory syndrome coronavirus-2 (SARS), which spreads by close contact and aerosols. The anesthesiologist is particularly vulnerable to aerosols while performing intubation and other airway related procedures. Regional anesthesia (RA) minimizes the need for airway manipulation and the risks of cross infection to other patients, and the healthcare personnel. In this context, for prioritizing RA over general anesthesia, wherever possible, a structured algorithmic approach is outlined. The role of percentage saturation of hemoglobin with oxygen (oxygen saturation), blood pressure and early use of point-of-care ultrasound in differential diagnosis and specific management is detailed. The perioperative anesthetic implications of multisystem manifestations of COVID-19, anesthetic management options, the scope of RA and considerations for its safe conduct in operating rooms is described. An outline for safe and rapid training of healthcare personnel, with an Entrustable Professional Activity framework for ascertaining the practice readiness among trained residents for RA in COVID-19, is suggested. These are the authors' experiences gained from the current pandemic and similar SARS, Middle East Respiratory Syndrome and influenza outbreaks in recent past faced by our authors in Singapore, India, Hong Kong and Canada.
\end{abstract}

\section{INTRODUCTION}

COVID-19 caused by the severe acute respiratory syndrome coronavirus-2 (SARS-CoV2) is highly contagious (basic reproduction number, R0: 2-2.5), and has spread rapidly and overwhelmed the healthcare capacities. ${ }^{12}$ This has created unprecedented disruptions in global medical supply chains and has resulted in cessation of elective surgeries. We state the considerations of COVID-19 pandemic on regional anesthesia (RA) practice, provide algorithmbased scheme for prioritizing RA for management of patients scheduled for surgery. We also outline strategies to prevent infection among the healthcare personnel and nosocomial spread based on our experiences from the current COVID-19 and previous swine influenza pandemics, avian SARS and Middle East Respiratory Syndrome (MERS) outbreaks.

\section{PERIOPERATIVE IMPLICATIONS}

The virus

The SARS-CoV2 is a beta coronavirus that is enveloped, unsegmented, positive single stranded
RNA virus with $79.5 \%$ and 50\% resemblance to SARS-CoV and Middle East Coronavirus, respectively. $^{3}$ The median incubation time for COVID-19 is 5.1 days. Evidence shows the evolution of two viral genomes with $\mathrm{L}$ type that is aggressive corresponding to the outbreak in china and $S$ type with milder illness but higher risk of transimission. ${ }^{34}$ Majority of manifestations are related to the affinity of the virus to membrane bound peptidase ACE2 present in lung and other tissues, thereby acting as functional receptor for the virus.

\section{Epidemiology, clinical features, investigations}

The COVID-19 outbreak was initially reported in Wuhan in China and subsequently declared a pandemic in March 2020. The countries with previous exposure to other coronavirus epidemics such as SARS, MERS and influenza virus (HINI) have effectively contained the infection after an initial spike with lower number of deaths and fewer healthcare worker infections. ${ }^{6}$

Clinical features of COVID-19 include history suggestive of respiratory infections, such as fever, cough, with or without expectorations, myalgia, sore throat and atypical manifestations, such as anosmia, dermatological lesions, ocular manifestations, hypertensions, stroke and acute coronary events. ${ }^{78}$ Multisystem manifestations are dependent on rapidity of viral replication and host immune responses. The presence of ACE2 downregulation or shedding results in dysfunction of renin-angiotensin system and increased pulmonary vascular permeability, while overactivation of $\mathrm{T}$ cells and antibody dependent enhancements result in systematic inflammatory response, cytokine storm, adult respiratory distress syndrome (ARDS), multiorgan dysfunction syndrome and death. ${ }^{4}$

Pertinent investigations include full blood count for knowing the white cell function, degree of immunosuppression, preoperative hemoglobin, bleeding and clotting functions, renal function and cardiac evaluations, such as ECG and echocardiography that are performed as required. Chest X-ray could show posterobasal pathology that progress to ground glass opacities and diffuse lung pathology in severe illness. ${ }^{9}$ CT scans and advanced chest imaging modalities are reserved for occasions when the diagnosis is not clear between hypoxia of pulmonary parenchyma pathology and or inflammatory response and microthrombi in pulmonary vasculature.

Confirmation of COVID-19 is through nucleic acid tests like real-time reverse transcription 
polymerized chain reactions ( $\mathrm{rtPCR} ;<5 \%$ false negative). Rapid serological tests with $\operatorname{IgM}$ and $\operatorname{IgM}$ antibody testing have not been consistent with high incidence of false negatives $(10 \%-$ $15 \%){ }^{1011}$ While planning for emergency surgeries in patients who are rtPCR negative and but still have high clinical index of suspicion can be considered for antibody testing. When resources are limited, the patients should be managed as 'clinical suspects' with full precautions as for COVID-19 confirmed cases.

\section{Spectrum of disease}

The spectrum of disease presentations is variable and majority of cases being mild (80\%) while 14\% have severe illness requiring oxygen therapy and $4 \%-10 \%$ need intensive care support with a case fatality rate of $1 \%-4 \% .{ }^{8}$ The percentage of critical illness and mortality tend to vary with the geographical area, socioeconomic status of presentations. Reports have shown the increasing incidence of non-ARDS presentations of hypoxia suggesting the presence of lung microvascular inflammatory response and need for caution. Asymptomatic careers from close contact of patients with COVID-19 are known to shed the virus for up to 2 weeks and the degree of infectivity is highest within the first week.

\section{Patient vulnerabilities}

COVID-19 is reported to be milder in children and the elderly are at risk with the degree of severity of illness being higher (14\%-20\%), requiring intensive care support. ${ }^{8}$ Pregnant mothers have moderate illness as compared with previous coronavirus outbreaks with vertical transmission being unlikely and data is evolving. ${ }^{9}$ Patients with comorbidities such as diabetes, lung disease and immunocompromise have higher incidence of severe systemic manifestations, critical care needs and fatalities. ${ }^{8} 1213$

\section{Transmission}

The chief mode of transmission is through droplets and it is known to occur among close contacts who are exposed to periods of exposure more than 30 minutes and within close proximities of less than 2 meters. Aerosol or air borne spread is described and the virus has been isolated through vents from rooms of infected patients. Spread to healthcare workers and nosocomial spread is reported to occur through droplets and through fomites. The virus has been isolated from doorknobs and by swabbing close proximity of patients for 2-3 weeks. ${ }^{14}{ }^{15}$ Water fountains, sinks and basins around patients have chiefly been the source. The resurgence of viral transmission has been noted through clusters or point source transmission in communities (eg, dormitories, poor socioeconomic status) where social distancing and fomite precautions were not adequately practiced. ${ }^{16}$

\section{Aerosolization and high-risk contact areas}

There are reports to suggest the chance of detection of the virus for periods of up to 2-3 weeks even during convalescence. Flushing of toilets is known to have caused aerosolization of the virus. ${ }^{15}$ This potential for fecoaerial route needs to be cautioned to all healthcare personnel handling patients in acute areas and in operating theaters when patients tend to defecate after sympathetic blockade from spinal subarachnoid block. Cleaning of patients and rolling of diapers need to be done with cover films for lower body and with minimum number of personnel within the operating rooms and in postoperative care locations.

\section{Protection in general}

Three levels of personal protection standards are described based on the mechanism and risk of spread. Fomites or contact precautions require fluid resistant gloves and gowns. Droplet precautions requires fluid resistant gloves, gowns, face shields or fluid-resistant face masks and goggles. Aerosol or airborne risk preventions include respirators such as $\mathrm{N} 95$ ( $\mathrm{N}$-not resistant to oil; 95\% protection against $0.3 \mu \mathrm{m}$ particles) or FFP2/3(European union standards: filtering face piece effective against $94 \%$ particles-FFP2 and 99\% particles-FFP3) and/or powered air purifying respirators (PAPR) in addition to all droplet precautions. ${ }^{17}$

The authors identify 'very high aerosol risks' when the trajectory of exhaled viral expulsions are higher when intubation is done during resuscitations, while handling restless patients with loud vocalizations or working in the vicinity of ill-fitting face helmets or noninvasive ventilation equipment with large leaks (eg, when hip fracture catheters are placed to achieve patient mobility for high-risk patients found to be unsuitable for immediate corrective surgery and are in high-dependency units with pressurized oxygen therapy)

\section{Communication within team with PPE donned}

Communications within the anesthesia team and among the theater personnel are known to be difficult, especially when teams wear advanced personal protection measures such as PAPR. Ways of communications within team must be worked out before donning on full PPE. These could include wireless simultaneous-talk communications systems that loops the anesthesia team, scrub team, surgeons, theater support and anteroom runner waiting outside the concealed OR. Simpler modes of communications like sterile white board or writing pads can be explored for resource limited locations. Mobile phones and other personnel communication systems are usually deposited at the ante-room for infection control purposes.

\section{ANESTHETIC IMPLICATIONS}

\section{Decision making for postponement or progression to OR}

During peak periods of the pandemic transmissions, the priority is to conserve the resources for the management of patients with emergencies, and those with cancer requiring emergent need for surgeries. Other surgeries need to be postponed and patients need to be counseled appropriately on the wisdom of this choice. These decisions need to be aligned with the national and health regulatory policies of the system and be dynamically revised based on the pandemic alert levels.

\section{Preoperative evaluation}

When patients are scheduled for surgery, a through preoperative evaluation, when possible, is advised. Chief principles are, in addition to routine preoperative risk stratifications, to assess the extent of COVID-19 disease severity, presence of end organ compromise, multiorgan dysfunction, level of hemodynamic stability. ${ }^{13}$ Fever, dehydration, poor oral intake and sepsis can present with altered fluid and electrolyte imbalance. Cardiovascular system evaluation to ascertain the presence of undiagnosed hypertension during COVID-19 that could result from ACE2 interactions is important. A 'false normal' blood pressure is a possibility in early sepsis or with intercurrent infections in the background of hypertension of COVID-19. There are multiple reports of intensive care patients with sudden deteriorations and hemodynamic downturn after 1-2 weeks of apparent stability, that tend to suggest this inherent peril. Cytokine storm has been reported in patients with severe manifestations of cardiovascular feature such as myocarditis. ${ }^{5}$ The level of host immune responses determine the degree of multisystem manifestations such cardiac failure, ARDS, liver and kidney failure and procoagulant states 


\begin{tabular}{|c|c|c|c|c|}
\hline System & Features & Investigation & Treatment & Comments \\
\hline CVS & $\begin{array}{l}\text { Palpitation } \\
\text { Hypertension } \\
\text { Myocarditis } \\
\text { Cardiomyopathy may result } \\
\text { from myocarditis, profound } \\
\text { systemic inflammation } \\
\text { and/or microvascular } \\
\text { dysfunction. }\end{array}$ & $\begin{array}{l}\text { ECG: diffuse ST-elevations } \\
\text { as seen in myopericarditis, } \\
\text { nonspecific ST changes, low } \\
\text { voltage in the limb leads, and } \\
\text { PVCs } \\
\text { Cardiac markers: } \\
\text { Troponin, NT Pro-BNP, CRP, } \\
\text { Ferritin, D-Dimer, IL-6, LDH } \\
\text { POCUS. }\end{array}$ & $\begin{array}{l}\text { Stable patients: guideline-directed medical } \\
\text { therapy for heart failure. } \\
\text { Patients with refractory shock: consider PAC to } \\
\text { help guide the use of inotropes, vasopressors } \\
\text { or mechanical support (ECMO). } \\
\text { Endomyocardial biopsy may have a role in } \\
\text { select patients being considered for clinical } \\
\text { trials of anti-inflammatory therapy. }\end{array}$ & $\begin{array}{l}\text { SARS Cov- } 2 \text { uses tissue ACE- } 2 \text { as receptor. } \\
\text { Vascular endothelium and myocardium is } \\
\text { affected thus. } \\
\text { SARS-CoV-2 can lead to immune cell } \\
\text { recruitment and myocarditis. } \\
\text { Released cytokines have the potential to } \\
\text { trigger cardiomyocyte dysfunction and } \\
\text { cardiac depression. } \\
\text { ACE inhibitors, ARBs upregulate expression } \\
\text { of ACE-2, usage may potentiate risks. }\end{array}$ \\
\hline RS & $\begin{array}{l}\text { Cough } \\
\text { Pneumonitis } \\
\text { ARDS }\end{array}$ & $\begin{array}{l}\text { CT chest: ground glass opacities- } \\
\text { typically bilateral and peripheral, } \\
\text { Ground glass opacities } \\
\text { consolidations or cordlike } \\
\text { opacities, } \\
\text { 'reverse halo', } \\
\text { 'crazy-paving'. }\end{array}$ & $\begin{array}{l}\text { Oxygen } \\
\text { Ventilation-NIV, invasive } \\
\text { ECMO } \\
\text { Airway clearance, bronchial hygiene. }\end{array}$ & $\begin{array}{l}\text { Increased pulmonary vascular permeability } \\
\text { due to increased production of angiotensin } \\
\text { II. } \\
\text { Two type of presentations of hypoxia: } \\
\text { ARDS with poor lung compliance } \\
\text { Non-ARDS inflammatory pulmonary } \\
\text { microvascular disease-good lung } \\
\text { compliance. }\end{array}$ \\
\hline
\end{tabular}

\begin{tabular}{|c|c|c|c|c|}
\hline Hemat & $\begin{array}{l}\text { Leukopenia, Lymphopenia } \\
\text { Thrombocytopenia } \\
\text { Microthrombi } \\
\text { DIC } \\
\text { Covid-19-associated } \\
\text { coagulopathy (CAC). } \\
\text { Antiphospholipid antibody } \\
\text { syndrome like combined } \\
\text { artero venous thrombo- } \\
\text { inflammation }\end{array}$ & $\begin{array}{l}\text { CAC: } \\
\text { Elevations in fibrinogen and } \\
\text { D-dimer levels with parallel rise } \\
\text { in markers of inflammation (eg, } \\
\text { CRP). }\end{array}$ & $\begin{array}{l}\text { Supportive. } \\
\text { Blood component therapy should be reserved } \\
\text { for those with active bleeding, requiring an } \\
\text { invasive procedure, or who are otherwise at } \\
\text { high risk for bleeding complications. }\end{array}$ & $\begin{array}{l}\text { Thrombocytopenia may be caused by } \\
\text { reduced production, increased destruction, } \\
\text { and increased consumption } \\
\text { Unlike classic DIC, the degree of aPTT } \\
\text { elevation is often less than PT elevation } \\
\text { (likely due to increased factor VIII), the } \\
\text { thrombocytopenia is mild (platelet count } \\
\left.\sim 100 \times 10^{9} / \mathrm{L}\right) \text {, and microangiopathy is rare. }\end{array}$ \\
\hline Renal & $\begin{array}{l}\text { Renal injury } \\
\text { Prerenal failure? } \\
\text { Azotemia? }\end{array}$ & $\begin{array}{l}\text { Proteinuria } \\
\text { Hematuria } \\
\text { Acute kidney injury scoring. }\end{array}$ & $\begin{array}{l}\text { Diuretics, } \\
\text { Dialysis/CRRT using HCO/MCO membrane, } \\
\text { endotoxin removal by polystrene fibers } \\
\text { functionalized with polymyxin B1. }\end{array}$ & $\begin{array}{l}\text { Effect on kidneys via- } \\
\text { cytokine damage, organ cross-talk and } \\
\text { systemic effects of fluid overload, third } \\
\text { spacing, hypotension, endothelial damage, } \\
\text { rhabdomyolysis, endotoxins. }\end{array}$ \\
\hline CNS & $\begin{array}{l}\text { Headache, dizziness, } \\
\text { impaired consciousness, } \\
\text { acute cerebrovascular } \\
\text { problems. }\end{array}$ & $\begin{array}{l}\text { EEG } \\
\text { CSF study for virus } \\
\text { Imaging }\end{array}$ & Supportive & $\begin{array}{l}\text { Possibility of non-convulsive status } \\
\text { epilepticus should be ruled out in all critical } \\
\text { patients with change on mental status. }\end{array}$ \\
\hline GIT & $\begin{array}{l}\text { Anorexia, nausea, vomiting, } \\
\text { diarrhea, abdominal pain, } \\
\text { bleeding. }\end{array}$ & Fecal RT-PCR for SARS-COV-2. & Supportive & $\begin{array}{l}\text { SARS-CoV-2 enters gastrointestinal epithelial } \\
\text { cells, and the feces of COVID-19 patients are } \\
\text { potentially infectious. Fecal excretion may } \\
\text { persist after sputum becomes negative in } \\
\text { patients for } 1-11 \text { days. }\end{array}$ \\
\hline General & $\begin{array}{l}\text { Sepsis, multiorgan failure } \\
\text { Anosmia } \\
\text { Petechial rash } \\
\text { Papular de squamation. }\end{array}$ & $\begin{array}{l}\text { RT-PCR from nasopharyngeal } \\
\text { swab, CT scan chest, } \\
\text { inflammatory markers. }\end{array}$ & $\begin{array}{l}\text { Supportive- systemic } \\
\text { antiviral/hydroxychloroquin/Immunotherapies } \\
\text { (anti IL-6) } \\
\text { Surviving sepsis guidelines. }\end{array}$ & $\begin{array}{l}\text { Cytokine release syndrome, cytokine storm } \\
\text { causing fever and multiorgan dysfunction } \\
\text { Topical anti-inflammatory drugs. }\end{array}$ \\
\hline Anti viral Therapy & $\begin{array}{l}\text { QTc prolongations } \\
\text { Arrythmias } \\
\text { Hepatic injury } \\
\text { Renal injury. }\end{array}$ & $\begin{array}{l}\text { ECG } \\
\text { Liver and renal function tests. }\end{array}$ & $\begin{array}{l}\text { Minimize or use with caution medications that } \\
\text { cause further worsening } \\
\text { Concerns with coadministrations of } \\
\text { ondansetron and antibiotics. }\end{array}$ & $\begin{array}{l}\text { Assess disease severity } \\
\text { Correlate with antiviral side effects } \\
\text { Decide anesthesia plan accordingly. }\end{array}$ \\
\hline
\end{tabular}

ACE, angiotensin converting enzyme; aPTT, activated partial thromboplastin time; ARB, angiotensin receptor blocker; ARDS, adult respiratory distress syndrome; CNS, central nervous system; CRP, C reactive protein; CRRT, continuous renal replacement therapy; CSF, cerebrospinal fluid; CVS, cardio vascular system; DIC, disseminated intravascular coagulation; ECG, electrocardiogram; ECMO, extracorporeal membrane oxygenation; EEG, electro encephalogram; GIT, gastrointestinal tract; HCO/MCO, high cut-off/ medium cut-off; IL-6, interleukin-6 ; LDH, lactate dehydrogenase; NIV, non-invasive ventilation; NT Pro-BNP, N terminal pro hormone brain natriuretic peptide; POCUS, point-of-care ultrasound; PT, prothrombin time; PVC, premature ventricular complex; RS, respiratory system; RT-PCR, reverse transcription polymerase chain reaction; SARS-COV-2, severe acute respiratory syndrome coronavirus-2.

that results in microthrombi requiring systemic anticoagulation. ${ }^{13} \mathrm{~A}$ detailed list of organ system concerns and preoperative considerations are stated in table 1.

The presence of concomitant antiviral therapy and possibility of side effects and drug interactions should be considered. Chloroquine and hydroxychloroquine have risk of QTc prolongations in combinations with azithromycin, torsades de pointes, sudden cardiac death and acute renal failure. ${ }^{18}$ Patients with other antiviral therapy (favipiravir, lopinavir/ritonavir, nitazoxanide, ivermectin and interferon) need to be rigorously evaluated for evidence of abnormal liver and renal function, psychiatric symptoms and exacerbations of gastrointestinal manifestations. ${ }^{18} 19$

\section{Rationing resources: impact on scarce resources such as intensive care unit beds}

Considerations to provide RA as sole anesthetic over general anesthesia (GA) could minimize need for postoperative care 


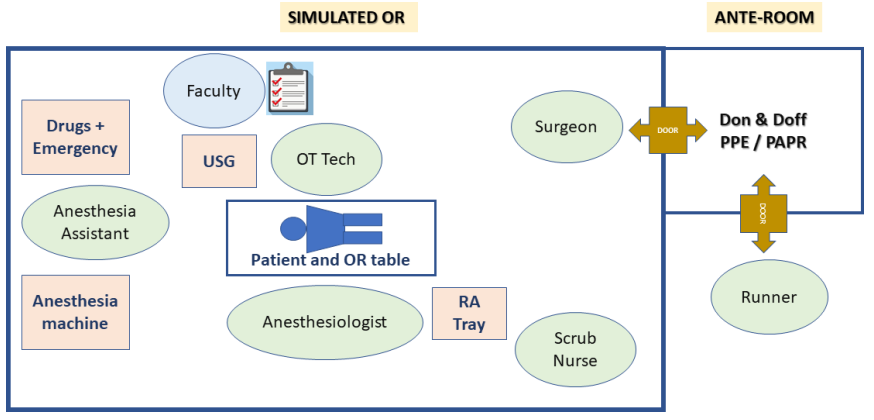

Figure 1 In situ simulation training layout showing the organization of simulated training in operation room with regional anesthesia set up and infection control practices training. OR, operating room; PAPR, powered air purifying respirators; RA, regional anesthesia.

in intensive care and high-dependency units that could preferentially be allocated for patients with severe illness. These resources include physical acute care beds and care locations, ventilation equipment, acute care advanced monitoring capabilities and trained personnel that could support them.

\section{Conservation of PPE}

With global disruption in supply chains and increased demands in the light of the pandemic, reserves for PPE and other consumables have been extremely short. In the authors institute, PPE is rationed to one per day per person and use of protective shields and splash proof layers are encouraged to protect n95 from being contaminated and simple surgical masks are worn over. Non-aerosol generating procedures and resource-limited settings could consider ways of preserving PPE and other equipment usage. Resterilizations of respirators have been reported to be achieved with UV-C light with wavelength between 100 and $280 \mathrm{~nm}$ with total dose more than $1 \mathrm{Jcm}^{-1}$. These are achieved through biosafety cabinets that are mainstay in laboratories that are presently not functional due to lockdown. The contact period needed is $62 \mathrm{~min}$ to 4.3 hours per side for respirators and 20 min per side for face shields and a maximum of 20 times of resterilizations is recommended. ${ }^{20}$

\section{Creation of COVID teams}

The chief aims of manpower reallocation and segregation to teams is to ensure that frontline staff are not overworked and exposed to mental and physical exhaustion. Anesthesia and perioperative care services need to be segregated and rotated once in 2 weeks for uniform distribution of workloads and appropriate rationing of rest periods. A minimum of three teams, where possible, are made when elective surgeries are canceled. First team is allocated the continuing care of cancer and other emergency surgeries and management of perioperative care services. Second team is assigned to intensive care units (ICUs) and other high-dependency units that have the capacity to ramp up to function as mechanical ventilation supported care areas. Third team covers outpatient, inpatient and virtual consult services, labor analgesia and acute and chronic pain management. Where the system is not overwhelmed, there must be provisions for allowing rapid code training, PPE/PAPR training, and simulation for orientation for non-intensivists to remain updated so that they are ready to support ICU manpower needs.

\section{Provider training and simulation \\ Donning and doffing of PPE}

The healthcare personnel need to be familiarized on the types of PPE, PAPR and be imparted with training on effective donning and doffing with trained observers who provide feedback. This helps in judicious and appropriate use of supplies for the right clinical requirements and reduction of self-contamination of healthcare personnel.

\section{Rapid code team training}

Protected code blue simulation of steps and sequences while caring for patients with COVID-19 needs to be considered in every healthcare location that manage these highly infectious patients. The authors have reported rapid cohort and workflow improvements within the first few sessions. ${ }^{21}$ These workflow improvements were attributed to periodic updates, morning huddles and structured debriefing sessions that discuss the common gaps, expected standard of care and opportunities for improvement. The actual time taken for code activation to PPEdonned CPR readiness is known to be improved to just about 4 min. ${ }^{21}$ A RA operating room (OR) simulation set up, the staff observing, the participants and confederates' roles and the layout are shown in figure 1.

RA crisis scenarios (patient collapse, respiratory compromise, local anesthetic systemic toxicity) that include rapid control of airway and cardiopulmonary resuscitation with PPE in place are be practiced. The focus is on the efficiency of the resuscitation, effective team communication, prevention of cross contaminations and adherence to infection control measures that are in vogue.

An entrustable professional activity (EPA) framework for readiness of a trained resident in performing a regional anesthetic in patients with COVID-19 is stated in table 2. While aerosol risky clinical situations are not the instances for residency training, senior residents that are 'practice ready' need to be groomed for taking up leadership roles with RA care when the system is overwhelmed.

\begin{tabular}{|c|c|c|}
\hline S no & Domain & EPA \\
\hline 1 & Infection control & Demonstrates proficiency in donning and doffing of PPE \\
\hline 2 & Crisis management and infection control & Demonstrates adequacy of resuscitation skills in simulated protected codes \\
\hline 3 & Communication & Demonstrates effective communication and coordination within protected code teams \\
\hline 4 & System based management & Demonstrates understanding of pathophysiology and multisystem concerns of COVID-19 \\
\hline 5 & Regional anesthesia & Demonstrates ability to perform regional anesthesia procedures with remote supervision \\
\hline 6 & Crisis management and Regional anesthesia & Demonstrates ability to recognize and manage regional anesthesia crisis in simulation \\
\hline 7 & Critical thinking & Demonstrates appropriate decision-making in patient disposition and safe transfer \\
\hline
\end{tabular}

An EPA guided framework for suitability of trained residents in performing regional anesthesia in COVID-19. The domains include regional anesthesia, crisis management, infection preventions and team management skills and critical thinking.

EPA, entrustable professional activity. 


\section{Utility of point of care ultrasound}

Point-of-care ultrasound (POCUS) provides valuable and scalable information about patients' cardiovascular and pulmonary status which can guide the anesthetist on the conduct of safe anesthetic. Important parameters evaluated through POCUS that provide valuable information for decision making for anesthesia include ${ }^{22}$ :

- Lung ultrasound: consolidation implies pneumonia, whereas Kerley B lines ( $>3$ ) suggests pulmonary edema and lung points are useful in early diagnosis of pleural effusion.

- Inferior vena cava (IVC) filing status: flattened out IVC implies hypovolemia resulting from dehydration, septic vasodilatation and distributive shock.

- Transthoracic echocardiogram: global hypokinesia and regional wall motion abnormalities (RWMA) might suggest septic cardiomyopathy or viral myocarditis; right ventricular dilatation would suggest pulmonary embolism.

The presence of lung pathology, cardiovascular compromise and hypovolemia that is identified through POCUS helps in anesthesia risk stratification, counseling, decision for regional vs GA and for planning postoperative disposition and level of monitoring. ${ }^{13} 1922$

\section{ANESTHETIC MANAGEMENT}

Choice of anesthetic

When surgery is indicated as an emergency, patients need to be stratified as for regional and general anesthesia based on their levels of oxygenation.

- A patient with low oxygenation levels presenting with a percentage saturation of hemoglobin with oxygen $\left(\mathrm{SpO}_{2}\right)$ less than $93 \%$ has a high probability of further deterioration over the course of time and should be considered for early airway control and stabilization with endotracheal intubations. $^{19}$

- Patients with better oxygenation status should be considered for RA when surgery can be done solely under RA. ${ }^{23}$

\section{Prioritizing RA over GA}

The conduct of RA, when not contraindicated, could possibly help in conserving supplies that are scarce during pandemics. These include drugs for performing general anesthetics, systemic analgesics, airway support measures used intraoperatively and postoperatively, use of portable oxygen supplies, use of high dependency and acute care support areas (space and trained personnel). The total reduction in cost with regional anesthetics is known to be $20 \%$ when compared with general anesthetics. ${ }^{24}$

Pulmonary presentations of COVID-19 such as consolidation, diffuse lung disease and inflammatory pathology, result in poor oxygenation, impaired gas exchange, irritable airways. These result in copious secretions that might pose higher risk of airway reactivity with higher incidence of intraoperative and postoperative complications. RA affords the sparing of airways and reduction of these complications. ${ }^{23}$ Case series of patients with COVID-19 have shown the safety of performing the entire procedure under RA with patients' face protected with simple face masks. ${ }^{25}$ No healthcare worker infections were noted from patients with surgeries done under RA. Neurological sequelae such as meningitis and encephalitis from central neuraxial blockade were not reported. Patients with pulmonary pathology that have excessive cough, inability to lie supine and/or dyspneic, with inability to sustain $\mathrm{SpO}_{2}$ more than 92\%, should be safely considered for GA and intubated under controlled conditions at the start with full PPE or PAPR donned.

\section{Scope of RA and cancer surgery}

Emergency orthopedic operations, lower abdominal and pelvic operations such as cesarean section, ectopic pregnancy, strangulated hernias, ovarian cysts torsion, appendicectomy, lower abdominal and colorectal operations, perineal and urological operations can be performed under RA. Among cancer surgeries, primary breast cancer surgery, simple mastectomy and sentinel lymph node biopsy and modified radical mastectomy can be performed with multilevel thoracic paravertebral block. Upper and lower extremity orthopedic cancer surgery (humeral, femoral and tibial surgery) can be conducted under peripheral nerve blocks (PNB) and central neuraxial blocks (CNB). Supplemental sedation should be judiciously employed along with RA. When a combination of regional and GA is required, administration of regional anesthetics help to conserve and negate the use of sedatives and analgesics enhancing the scope for maintaining the reserves.

\section{Regional anesthesia}

Systematic approach to RA

On stratification of patient for RA the authors recommend early use of POCUS for diagnostic accuracy and for therapeutic purposes. Patients with deteriorating oxygenation levels or dyspneic with $\mathrm{SpO}_{2}$ levels lower than 93\% should be safely proritized for early airway support and GA (figure 2). Three broad presentations are known. First, normotensive patients with no evidence of sepsis and with good cardiac function have limited contraindications for central or PNBs. Normotensive patients with poor oxygenation tend to have primary pulmonary causes of hypoxia and would benefit by early airway support and intensive care. Hypotensive patients need to be categorized into cardiogenic and septic causes or they could present with mixed picture. The addition of non-invasive cardiac output (CO) measures such as pulse contour analysis obtained from peripheral intra-arterial lines would provide vital information on the systemic vascular resistance (SVR) and CO measurements. These are considered when resources permit. Second, cardiogenic causes such as cardiomyopathy and viral myocarditis present with global hypokinesia, RWMA with normal CO and normal SVR. ${ }^{22}$ These patients require fluid restriction and inotropes are initiated early to augment cardiac contractility. Finally, hypotensive patients with sepsis, show hyperdynamic cardiac activity with good CO and markedly reduced SVR. These patients benefit from fluid challenges and vasopressors and PNBs are preferred. Patients with frank sepsis, with unstable hemodynamics and deteriorating systemic oxygenations might benefit from early ICU admissions, mechanical ventilatory support.

The use of non-invasive ventilation with high flow nasal cannula, full face helmets, though known to be safely administered, are mostly reserved for set ups with prior training, those with experience in managing aerosol generating infectious cases and in the presence of negative pressure rooms with isolation and attending staff wearing full PPE. ${ }^{9}$ When oxygenation is refractory to these measures extracorporeal membrane oxygenation (ECMO) might need to be initiated. ${ }^{26}$

\section{CNB versus PNB or sedation}

\section{When to consider CNB?}

Central neuraxial blockade should be considered in the absence of sepsis wherever admissible. The presence of multiorgan dysfunction, coagulopathy, thrombocytopenia, therapeutic anticoagulation for micro thrombi and frank sepsis are contraindications for central neuraxial blockade. A low-dose spinal anesthetic 


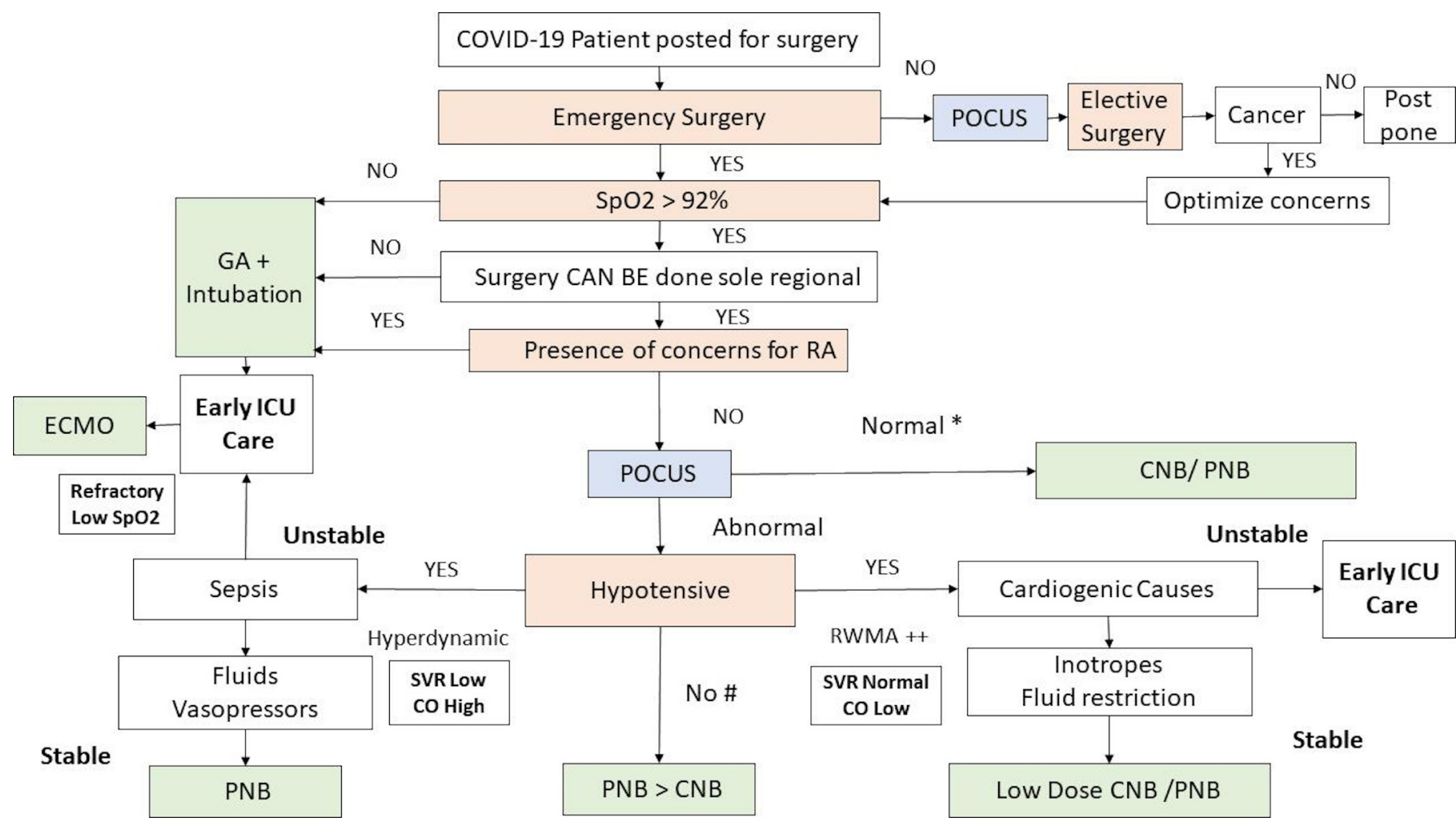

Figure 2 Showing the systematic approach for considering regional anesthesia in COVID-19 patients. *Hypertension is a common presentation in COVID-19 due to the interactions of the virus with the angiotensin converting enzyme (ACE2): a false normal blood pressure could be early signs of deterioration in a previously hypertensive COVID-19 presentation a.\# Sepsis with white cell count $>15 \times 10^{\wedge} 9 / \mathrm{L}$ are relative contraindications for CNB, COVID-19 patients might present with lymphopenia and cardinal manifestations of sepsis may not be seen. CNB, central neuraxial block; CO, cardiac output; ECMO, extra corporeal membrane oxygenation; GA, general anesthesia; POCUS, point of care ultrasound that includes transthoracic echocardiography (TTE), lung ultrasound and IVC scan for volume status; PNB, peripheral nerve block; Sp02-percentage oxygen saturation of hemoglobin; SVR, systemic vascular resistance when measured by non-invasive pulse contour analysis through peripheral arterial pressure.

can be administered in patients with early sepsis (white cell count $<15 \times 10^{\wedge} 9 / \mathrm{L}$ ), who have a left ventricular ejection fraction more than $35 \%$ and whose blood pressure has been stabilized with intravenous fluids and vasopressors. ${ }^{23}$

\section{Role of PNB}

PNBs provide effective surgical plane of anesthesia with minimum change is systemic hemodynamics. Surgeries of the extremities and chest wall can be performed solely without general anesthetics (refer above). Regional anesthetics that might compromise the functioning of the diaphragm such as the interscalene and supraclavicular brachial plexus should be considered with caution in patients with lung compromise. The use of ultrasound might help in providing accurate dose and reduced volume of local anesthetics and can minimize complications.

Sedation when considered in regional anesthesia, might be used judiciously and closely monitored to minimize respiratory depression and hemodynamic compromise. A side stream endtidal carbon dioxide monitoring with suitable filters should be used when sedation is provided to patients. ${ }^{25}$

\section{Obstetric considerations}

Labor epidural analgesia is a specialty service that needs to be orchestrated well in a patient with COVID-19 who is actively laboring. The forceful exhalation from vocalization and expression of pain and hyperventilation and the subsequent aerosolization can make the use of simple face masks ineffective in preventing COVID-19. This mandates all healthcare personnel attending to these patients to wear full PPE. ${ }^{9}$ A coordinated approach for performing the epidural early in labor is recommended. ${ }^{9}$

Elective cesarean surgery could possibly be performed with CNB unless contraindicated. For emergency cesarean deliveries RA should be considered if $\mathrm{SpO}_{2}$ is $93 \%$ or above. Where an epidural catheter is in place, administration of alkalinized lignocaine $1.5 \%$ with $8.4 \%$ sodium bicarbonate will provide rapid operating conditions, and rapid onset $(3.5 \mathrm{~min})$ comparable to spinal subarachnoid blockade. Rapid sequence spinal anesthesia with the one touch technique could be considered if no epidural catheter is insitu. ${ }^{27}$ The act of performing a spinal anesthetic can pose risks to theater personnel and all need to wear full PPE.

When GA is needed for cesarean surgeries, extubation has a higher chance of aerosolization and cross contaminations more than during intubation. This is because, intubation is usually done rapid sequence with the patient paralyzed and with the cough suppressed from induction and paralysis.

\section{Prevention of infection during RA Administration of RA with barrier precautions}

When patients with COVID-19 are scheduled for surgery, it should possibly be performed in designated negative pressure operating rooms that have more than 12 air cycles per hour. ${ }^{28}$ All additional equipment and utilities from the room need to be evacuated with only a set of prepacked RA drugs, equipment, monitors and resuscitation supplies kept inside the room. The patient should be wearing simple face mask or oxygen mask 
to reduce the volume of droplets. All theater personnel should wear full PPE that include droplet protection grade face shields, goggles, splash proof N95 or FFP2/3 face masks, full length gowns, double gloves and shoe covers.

While performing the regional anesthetic, supporting theater personnel and proceduralist need to be protected with additional measures like having a protective plastic film interface, turning the patient to face away and by performing the procedure in lateral position instead of being supine or sitting, where possible. Surgical and scrub teams should be kept to minimum until the procedure is completed. Disposable consumables need to be considered so that decontamination is simpler.

\section{Decontamination after $R A$}

Once a regional anesthetic procedure is completed, surface decontamination of RA equipment and environment are to be conducted meticulously. The common high-risk areas of contact and cross contaminations include ultrasound machine controls and power source, RA trolley, monitoring equipment, electronic controls for the patient bed and OR table, light switches, hand wash area and taps. Proven methods of cleansing include 5000 ppm of sodium dichloroisocyanurate for surface of equipment and 1000 ppm for floors. ${ }^{15}$ When reusable equipment is used, they need to be disinfected with more than $70 \%$ alcohol.

\section{General anesthesia}

Preventing droplet contamination during intubation/extubation

GA is needed in the context of RA for a planned combined procedure (GA+RA, eg, esophageal surgery), or as a rescue measure to supplement anesthetic depth, or in a crisis when patient has a cardiorespiratory compromise. The conduct of of GA is aerosol generating, owing to the presence of leaks from mask ventilation, positive pressure ventilation intraoperatively and from forceful exhalation during emergence and coughing. Disconnections of circuits is minimized to prevent aerosol generations and when necessary is achieved with clamps applied to tracheal tubes and fresh gas flows turned off to minimize spraying droplets in patients' vicinity. ${ }^{19} 28$

Intubating team might consider use of PAPR, when available, in addition to PPE for the procedure, provide adequate anesthetic depth for intubation and emergence (avoiding cough), perform modified rapid sequence intubations while minimizing mask ventilation and use of aerosol generating procedures such as jet ventilations, nebulized topicalization of airways, awake fiberoptic techniques. ${ }^{19} 28$ Use of plastic films and enclosed plastic shields are described. The process of extubation tends to be more uncontrolled than intubations that have stable paralyzed patients and all team members need to keep full PPE donned until patient is safely extubated and transferred out.

\section{OR hygiene}

The perioperative course of COVID-19 needs to be managed as per fomites, droplet and aerosol precautions and ramped up as required. While minimizing non-essential equipment is the key, adequate preplanning helps in minimizing contamination of clean supplies in anteroom. Appropriate cleaning and decontamination of equipment, surface, floor is performed as mentioned. Disposal of contaminated patient and surgical linen should comply with biohazard regulations of the institute. All theater personnel are directed to proceed by a coordinated exit procedure to specified shower and decontamination facilities after the safe transit of patient. All donning and doffing of PPE is carried out only in designated locations with biohazard waste disposal options and use of mandatory alcohol-based hand rubs before and after handling the PPE. Documentations when done through digital records are done through plastic film covered computer keyboards and screens, but when involves manual paper charting, that needs to be well thought after, when absolute measures for cross contamination are to be in place.

\section{Patient care and postoperative dispositions}

Immediate postoperative care is usually provided within the operating room until patients are hemodynamically stable and recovered from sedation with minimum requirement for supplemental oxygen. Step down locations need to be equipped with isolation rooms and facilities for oxygen therapy and aerosol precaution capabilities. Clear plans for management of sudden deteriorations and anticipated issues in the postoperative period need to be communicated to care teams. ${ }^{19}$ 28-30

\section{Patient transit and within hospital transfer}

Patients with COVID-19 need to be transferred in and out of OR with pre-evacuated pathways bypassing the usual holding areas to minimize cross contaminations. ${ }^{29}$ Use of designated concealed exits for aerosol prone patients must be practiced. The concept of traffic control bundling that has predesignated transit pathways for patients with COVID-19 and healthcare staff movement is advised to minimize healthcare worker cross infections. ${ }^{31}$

\section{Preparing for post-lockdown staged reopening of full operating services}

As healthcare teams globally restart clinical services and resume elective surgeries, we propose the following considerations:

- All elective surgical admissions need to be screened for COVID-19, or atleast when there is a suspicion through rtPCR and rapid detection tests. ${ }^{32}$

- COVID suspect: need to be re-considered for postponement.

- Post COVID-positive patients, need to be cleared by the infectious disease teams of residual disease concerns

- Consider 6 weeks for elective surgery postponement in view of:

- Reactive airways and copious secretions.

- Lung residual pathology and reduced functional reserves.

- Coagulation and other organ system dysfunction.

- All patients with history of COVID-19 need to have:

- Full blood count, platelet and coagulation function where required.

- Room air $\mathrm{SpO}_{2}$ measurement: for evidence of residual lung pathology.

- Designate intermediate postoperative care provisions, not to be mixed with general population in post-operative care area, especially the elderly.

- Where there are no such provisions, consider isolation to MRSA and contact precaution areas and staff could wear PPE for recovery and transit as required.

- Social distancing in clinics, preanesthesia consult clinics, minimizing patient visitors for inpatients and postoperative care areas and hospital services is paramount.

\section{CONCLUSION}

The multisystem considerations of COVID-19 and the stage of clinical progression or extent of disease need to be ascertained while making the choice on the type of anesthetic. The algorithm based on use of portable and basic clinical measurements such as $\mathrm{SpO}_{2}$, blood pressure and POCUS is recommended. Clinical 
decision making in line with this algorithm can possibly be beneficial for the patient, the healthcare professionals and the community at large.

RA should be preferred over GA, where possible, to minimize aerosol risks and cross contamination of other patients and healthcare workers. Choice of RA could potentially limit the use of scarce resources such as anesthetic drugs, consumables, acute care and intensive care facilities. Resource-limited settings should prioritize RA to preserve vital supplies, minimizing the saturation of acute care locations while reconsidering methods for reuse of PPE or to re-engineer it.

Contributors $B A$ and $A C$ conceived the idea and collaborated with BJS in developing the manuscript. MKK and VC provided guidance throughout the entire process. BA provided the perspective from Singapore, AC and BJS from India, MKK from Hong Kong and VC from Canadian perspectives.

Funding The authors have not declared a specific grant for this research from any funding agency in the public, commercial or not-for-profit sectors.

\section{Competing interests None declared.}

Patient consent for publication Not required.

Provenance and peer review Not commissioned; externally peer reviewed.

This article is made freely available for use in accordance with BMJ's website terms and conditions for the duration of the covid-19 pandemic or until otherwise determined by BMJ. You may use, download and print the article for any lawful, non-commercial purpose (including text and data mining) provided that all copyright notices and trade marks are retained.

ORCID iDs

Balakrishnan Ashokka http://orcid.org/0000-0001-9175-7479

Arunangshu Chakraborty http://orcid.org/0000-0002-0069-700X

Manoj Kumar Karmakar http://orcid.org/0000-0002-3674-2095

\section{REFERENCES}

1 World Health Organisation (WHO). Report of the WHO-China joint mission on coronavirus disease 2019 (COVID-19), 2020. Available: https://www.who.int/docs/ default-source/coronaviruse/who-china-joint-mission-on-covid-19-final-report.pdf [Accessed Mar 2016].

2 Liu Y, Gayle AA, Wilder-Smith A, et al. The reproductive number of COVID-19 is higher compared to SARS coronavirus. J Travel Med 2020;27. doi:10.1093/jtm/taaa021

3 Tang X, Wu C, Li X, et al. On the origin and continuing evolution of SARS-CoV-2. Natl Sci $\operatorname{Rev} 2020 ; 395$.

4 Jin Y, Yang H, Ji W, et al. Virology, epidemiology, pathogenesis, and control of COVID-19. Viruses 2020;12:372.

5 Zheng YY, YT M, Zhang JY, et al. COVID-19 and the cardiovascular system. Nat Rev Cardiol 2020;2020

6 Wong JEL, Leo YS, Tan CC. COVID-19 in Singapore-Current experience: critical global issues that require attention and action. JAMA 2020;2020. doi:10.1001/ jama.2020.2467

7 Sanchez A, Sohier P, Benghanem S, et al. Digitate papulosquamous eruption associated with severe acute respiratory syndrome coronavirus 2 infection. JAMA Dermatol 2020. doi:10.1001/jamadermatol.2020.1704

8 Wu Z, McGoogan JM. Characteristics of and Important Lessons From the Coronavirus Disease 2019 (COVID-19) Outbreak in China: Summary of a Report of 72314 Cases From the Chinese Center for Disease Control and Prevention. JAMA 2020;2020

9 Ashokka B, Loh M-H, Tan CH, et al. Care of the pregnant woman with COVID-19 in labor and delivery: anesthesia, emergency cesarean delivery, differential diagnosis in the acutely ill parturient, care of the newborn, and protection of the healthcare personnel. Am J Obstet Gynecol 2020. doi:10.1016/j.ajog.2020.04.005
10 Abbasi J. The promise and peril of antibody testing for COVID-19. JAMA 2020. doi:10.1001/jama.2020.6170

11 National University of Singapore. Saw Swee Hock school of public health. COVID-19 science report. Available: https://sph.nus.edu.sg/wp-content/uploads/2020/04/COVID19-Science-Report-Diagnostics-27-Apr.pdf [Accessed 3 May 2020].

12 Huang C, Wang Y, Li X, et al. Clinical features of patients infected with 2019 novel coronavirus in Wuhan, China. Lancet 2020;395:497-506.

13 Alhazzani W, Møller MH, Arabi YM, et al. Surviving sepsis campaign: guidelines on the management of critically ill adults with coronavirus disease 2019 (COVID-19). Intensive Care Med 2020:1-34.

14 van Doremalen N, Bushmaker T, Morris DH, et al. Aerosol and surface stability of HCoV-19 (SARS-CoV-2) compared to SARSCoV-1. medRxiv 2020.

15 Ong SWX, Tan YK, Chia PY, et al. Air, surface environmental, and personal protective equipment contamination by severe acute respiratory syndrome coronavirus 2 (SARSCoV-2) from a symptomatic patient. JAMA. 2020;323:1610.

16 Inglesby TV. Public health measures and the reproduction number of SARS-CoV-2. JAMA 2020.

17 Verbeek JH, ljaz S, Mischke C, et al. Personal protective equipment for preventing highly infectious diseases due to exposure to contaminated body fluids in healthcare staff. Cochrane Database Syst Rev 2016;2:CD011621.

18 Simsek Yavuz S, Ünal S. Antiviral treatment of COVID-19. Turk J Med Sci 2020;50:611-9.

19 Greenland JR, Michelow MD, Wang L, et al. COVID-19 infection: implications for perioperative and critical care physicians. Anesthesiology 2020. doi:10.1097/ ALN. 0000000000003303

20 Farrokhian VG, Ho E, King ES, et al. Uv sterilization of personal protective equipment with idle laboratory biosafety cabinets during the COVID-19 pandemic. medRxiv 2020.

21 Foong TW, ELS N, Khoo CWE, et al. Rapid training of healthcare staff for protected code blue in COVID-19 pandemic. Br J Anaesth 2020;1.

22 Zhang L, Wang B, Zhou J, et al. Bedside focused cardiac ultrasound in COVID-19 infection from the Wuhan EpiCenter: the role of cardiac point of care ultrasound (POCUS), limited transthoracic echocardiography and critical care echocardiography. J Am Soc Echocardiogr 2020.

23 Stundner $\mathrm{O}$, Memtsoudis SG. Regional anesthesia and analgesia in critically ill patients: a systematic review. Reg Anesth Pain Med 2012;37:537-44.

24 Chan VW, Peng PW, Kaszas Z, et al. A comparative study of general anesthesia, intravenous regional anesthesia, and axillary block for outpatient hand surgery: clinical outcome and cost analysis. Anesth Analg 2001;93:1181-4.

25 Uppal V, Sondekoppam RV, Landau R, et al. Neuraxial anaesthesia and peripheral nerve blocks during the COVID-19 pandemic: a literature review and practice recommendations. Anaesthesia 2020. doi:10.1111/anae.15105

26 MacLaren G, Fisher D, Brodie D. Preparing for the most critically ill patients with COVID-19: the potential role of extracorporeal membrane oxygenation. JAMA 2020:2020. doi:10.1001/jama.2020.2342

27 Kinsella SM, Girgirah K, Scrutton MJL. Rapid sequence spinal anaesthesia for category-1 urgency caesarean section: a case series. Anaesthesia 2010;65:664-9.

28 Cook TM, El-Boghdadly K, McGuire B, et al. Consensus guidelines for managing the airway in patients with COVID-19: guidelines from the difficult airway Society, the association of anaesthetists the intensive care Society, the faculty of intensive care medicine and the Royal College of anaesthetists. Anaesthesia 2020.

29 LK T, Ang LS, Foong TW, et al. What we do when a COVID-19 patient needs an operation: operating room preparation and guidance. Can J Anesth 2020;6.

30 Wax RS, Christian MD. Practical recommendations for critical care and anesthesiology teams caring for novel coronavirus (2019-nCoV) patients. Can J Anaesth 2020;67:568-76.

31 Schwartz J, King C-C, Yen M-Y. Protecting health care workers during the COVID-19 coronavirus outbreak -Lessons from Taiwan's SARS response. Clin Infect Dis 2020. doi:10.1093/cid/ciaa255

32 Uppal V, Sondekoppam RV, Lobo CA, et al. Practice recommendations on neuraxial anesthesia and peripheral nerve blocks during the COVID-19 pandemic, 2020. Available: https://esraeurope.org/wp-content/uploads/2020/04/ESRAASRA-COVID-19Guidelines-.pdf [Accessed 1 May 2020]. 